\title{
Original
}

\section{Relationship between Type D personality and dropout from dental treatment in middle-aged adults}

\author{
Tomotaka Kato ${ }^{1,2)}$, Shinsuke Mizutani ${ }^{3,4)}$, Yojiro Umezaki2), \\ Seiichi Sugiyama5), and Toru Naito ${ }^{2)}$
}

\author{
1)Department of Oral Health Science, School of Dentistry, University of Washington, Seattle, WA, USA \\ 2)Section of Geriatric Dentistry, Department of General Dentistry, Fukuoka Dental College, Fukuoka, Japan \\ 3) Section of Geriatric Dentistry and Perioperative Medicine in Dentistry, Division of Maxillofacial Diagnostic \\ and Surgical Sciences, Faculty of Dental Science, Kyushu University, Fukuoka, Japan \\ 4)OBT Research Center, Faculty of Dental Science, Kyushu University, Fukuoka, Japan \\ 5)Private Practice, Yachiyo, Japan
}

(Received March 2, 2018; Accepted July 26, 2018)

\begin{abstract}
Type D personality-the tendency toward combined negative affectivity and social inhibitionis a risk factor for coronary heart disease. Although Type D personality has been linked to low adherence to diabetic care, it is unclear whether it is related to adherence to dental therapy. Thus, study examined the relationship between Type $D$ personality and withdrawal from dental therapy. A web-based questionnaire was used to compare a treatment dropout group $(n=225)$ and maintenance group $(n=236)$. The questionnaire was designed to assess Type D personality traits, reasons for dropout, and respondent sociodemographic characteristics and attitudes. There were no significant differences in the sociodemographic characteristics of the two groups. However, the proportion of respondents with a Type $D$ personality was significantly larger in the dropout group than in the maintenance group $(P=0.04)$. The most common reasons for dental therapy dropout were "improvement of symptoms" $(46.2 \%)$ and "busy with business or study" (30.7\%). The most frequent attitude reported in the dropout group was negative
\end{abstract}

Correspondence to Dr. Tomotaka Kato, Department of Oral Health Science, School of Dentistry, University of Washington, 1953 NE Pacific Street, Seattle, WA 98195, USA

E-mail: tomotaka1182@yahoo.co.jp

doi.org/10.2334/josnusd.18-0068

DN/JST.JSTAGE/josnusd/18-0068 feelings toward dental practice. In conclusion, Type D personality might be related to dropout from dental therapy.

Keywords: Type D personality; dental therapy dropout; web-based research; treatment adherence.

\section{Introduction}

Many studies have investigated the relationship of periodontal disease with systemic diseases (1-5). Periodontal disease control is very important in oral and general health, and supportive periodontal therapy has a key role in controlling periodontal disease $(6,7)$. However, only $20-50 \%$ of individuals regularly undergo such therapy $(8,9)$, and many choose to discontinue dental treatment. Cessation of dental therapy was reported to be associated with high risks of periodontal disease development and exacerbation $(6,7)$. Thus, it is essential to understand the reasons and attitudes associated with dental therapy dropout.

Type D personality refers to the simultaneous presence of negative affectivity and social inhibition $(10,11)$. In recent years, Type D personality has been studied as a potential risk factor for cardiovascular disease (12). During the last two decades, numerous studies have examined the relationship between personality type and cardiovascular disease. Denollet reported that the mortality rate of coronary heart disease (CHD) patients with Type D personality was 4.1 times that of other CHD 
patients (13). Moreover, Type D personality was more strongly associated with CHD recurrence and mortality than were the classical CHD risk factors (sex, age, left ventricular ejection fraction, and triple-vessel disease) (14).

Type D personality is also related to drug compliance and treatment adherence (15-19), and with low adherence to diabetic care. Few studies have assessed the effects of Type D personality on dental outcomes and interventions. An association of Type D personality with periodontal status was reported (20); however, no study has evaluated the association of Type D personality with adherence to dental therapy. With the hope of improving periodontal disease prevention, the present study investigated patients who withdrew from dental therapy, to determine their attitudes and investigate the relationship between Type D personality and treatment adherence.

\section{Materials and Methods}

\section{Participants and ethical considerations}

The present study was performed from December 2016 through April 2017, and the actual web-based questionnaire assessment was conducted from January 20, 2017 through January 24, 2017. The respondents were adults older than 45 years who had registered with an internet research company (JustSystems Corporation, Tokyo, Japan). The participants were classified into two groups: the dropout group and maintenance group. The dropout group included respondents who had discontinued dental therapy during the previous 10 years and had not revisited a dental clinic since that time. The maintenance group included respondents who had continued to visit a dental clinic for regular check-ups and had visited a dental clinic at least once in the previous 1 year.

The sample size of this study was designed to achieve an $\alpha$ error of 0.05 and a power of 0.9. A previous study reported that $35.6 \%$ of individuals in a maintenance group and $51.6 \%$ of those in a dropout group had a Type D personality (16). The present study design therefore called for including more than 220 participants in each group.

The present study was approved by the Ethics Committee for Clinical Research at Fukuoka Gakuen (approval number: 324). The research company only analyzed the questionnaire results; the analysts had no access to participant identifying information.

\section{Questionnaire design}

Reasons for dental therapy withdrawal

The reasons for discontinuing dental therapy were analyzed in the dropout group. The respondents were asked to select their top three reasons from among the following list: "moved out of area", "distance and scheduling of outpatient clinic", "improvement of symptoms", "departure of attending clinic staff", "economic factors", "distrust of clinic staff", "fear of treatment", "busy with business or study", "childcare or eldercare problem", "diseases other than oral symptoms", and "others".

\section{Participant attitudes}

The attitudes of the respondents in both groups were analyzed, and the questionnaire items were based on those detailed in a previous study (21). The questionnaire comprised 10 items, and responses were given on a 5-point scale, from 1 (strongly agree) to 5 (strongly disagree); the total score ranged from 10-50. The average of each item was compared between the two groups.

\section{Assessment of Type D personality}

In both groups, Type D personality was evaluated by using the Type D Personality Scale (DS14) $(10,11)$. Participants were asked to rate their responses on a 5-point scale, from 0 (false) to 4 (true). The DS14 contains two subscales: negative affectivity ( 7 items; range 0-28) and social inhibition (7 items; range 0-28). Type $\mathrm{D}$ personality was defined as a score of 10 or higher on both subscales.

\section{Statistical analysis}

All statistical analyses were performed with the IBM SPSS Statistics software program (Ver. 22.0, IBM, Armonk, NY, USA). Patient attitudes and data on Type D personality were analyzed with the Mann-Whitney $U$ test; all other factors were analyzed with the $2 \times 2$ chisquare test. The significance level was set at $5 \%$.

\section{Participants}

\section{Results}

Selected sociodemographic characteristics of the study participants are shown in Table 1. At the end of enrollment there were 225 people in the dropout group and 236 people in the maintenance group, and no statistically significant differences were observed between these groups in sex, marital status, or presence of children. In addition, there were no statistically significant differences in relation to employment or residential factors (data not shown). With regard to number of interruptions in dental therapy in the dropout group, 161 respondents (71.6\%) reported 1-2 interruptions, 41 (18.2\%) reported 3-5 interruptions, and $23(10.2 \%)$ reported $\geq 6$ interruptions. 
Table 1 Sociodemographic characteristics of respondents in the dropout and maintenance groups

\begin{tabular}{lccc}
\hline & Dropout & Maintenance & $P$ value \\
\hline No. of respondents & 225 & 236 & 0.83 \\
Male/Female & $115 / 110$ & $123 / 113$ & 0.37 \\
Age (years) & $56.3 \pm 7.0$ & $56.6 \pm 7.4$ & 0.79 \\
Married/Single & $177 / 48$ & $188 / 48$ & 0.19 \\
Children, yes/no & $165 / 60$ & $160 / 76$ & \\
\hline
\end{tabular}

Mann-Whitney $U$ test.

Table 2 Reasons for withdrawal from dental therapy

\begin{tabular}{lccc}
\hline & No. $(\%)$ & Type D (no.) & Non-Type D (no.) \\
\hline Improvement of symptoms & $104(46.2)$ & 76 & 28 \\
Busy with business or study & $69(30.7)$ & 42 & 27 \\
Economic factors & $33(14.7)$ & 24 & 9 \\
Moved out of area & $28(12.4)$ & 20 & 8 \\
Distrust of clinic staff & $26(11.6)$ & 20 & 6 \\
Distance and scheduling of outpatient clinic & $22(9.8)$ & 17 & 5 \\
Fear of treatment & $15(6.7)$ & 14 & 4 \\
Diseases other than oral symptoms & $10(4.4)$ & 6 & 4 \\
Departure of attending clinic staff & $6(2.7)$ & 2 & $5 \%$ \\
Childcare or eldercare problem & $6(2.7)$ & 1 & 6 \\
Others & $9(4.0)$ & 3 & \\
\hline
\end{tabular}

※ $2 \times 2$ chi-square test $P<0.05$ (Type $\mathrm{D}$ vs Non-Type $\mathrm{D}$ persons)

Table 3 Respondent attitudes

\begin{tabular}{lccc}
\hline & $\begin{array}{c}\text { Dropout } \\
n=225\end{array}$ & $\begin{array}{c}\text { Maintenance } \\
n=236\end{array}$ & $P$ value \\
\hline I am health conscious & $2.26(0.95)$ & $2.20(0.99)$ & 0.349 \\
I want to live an active life & $3.39(1.11)$ & $3.09(1.23)$ & $0.009 \%$ \\
I feel comfortable with the staff & $2.97(0.82)$ & $2.74(0.93)$ & $0.005 \%$ \\
I feel the treatment fee is expensive & $1.96(0.88)$ & $2.11(0.92)$ & 0.119 \\
I do not suffer from the distance or scheduling of the clinic & $2.46(1.09)$ & $2.20(1.09)$ & $0.006 \%$ \\
I think that dental examinations include oral x-rays and a periodontal test & $2.33(0.84)$ & $2.10(0.83)$ & $0.003 \%$ \\
I will visit the dentist with my family & $3.14(1.07)$ & $2.67(1.08)$ & $<0.001 \%$ \\
I think regular visits to the dental clinic are important & $2.31(0.85)$ & $1.69(0.77)$ & $<0.001 \%$ \\
The image I associate with dental practice is "neat", "kind" or "refreshing" & $2.64(0.86)$ & $2.31(0.88)$ & $<0.001 \%$ \\
The image I associate with dental practice is "fear" or "pain" & $2.51(1.02)$ & $2.78(1.17)$ & $0.028 *$ \\
\hline
\end{tabular}

Data presented as mean (standard deviation). * Mann-Whitney $U$ test $P<0.05$

\section{Reasons for dental therapy withdrawal}

Table 2 shows the reasons for dental therapy withdrawal. The most common reason was "improvement of symptoms" (46.2\%), followed by "busy with business or study" (30.7\%); other reasons included "economic factors" (14.7\%), and "moved out of area" (12.4\%) (the respondents were asked to indicate their top three reasons). In addition, although the reasons for dropout were similar for respondents with and without Type D personality, there was a significant difference between groups in those reporting a "childcare or eldercare problem".

\section{Participant attitudes}

Participant attitudes toward dental treatment are shown in Table 3. Statistically significant differences were observed between the dropout and maintenance groups for nearly all the items. In particular, the numbers of respondents reporting that, "I will see the dentist with my family", "I think it is important to visit a dental clinic regularly", and "The image I associate with dental practice is 'neat', 'kind' or 'refreshing' ", significantly differed $(P<0.001)$. However, there was no statistical difference in those reporting that "I am health conscious" or "I feel that treatment is expensive". 
Table 4 Association of Type D personality with withdrawal from dental therapy

\begin{tabular}{lccc}
\hline & Type D & Non-Type D & Total \\
\hline Dropout group & $152(52.4 \%)$ & $73(42.7 \%)$ & 225 \\
Maintenance group & $138(47.6 \%)$ & $98(57.3 \%)$ & 236 \\
\hline Total & 290 & 171 &
\end{tabular}

Table 5 Details of DS14 items

\begin{tabular}{|c|c|c|c|}
\hline & $\begin{array}{c}\text { Dropout } \\
n=225 \\
\end{array}$ & $\begin{array}{c}\text { Maintenance } \\
n=236\end{array}$ & $P$ value \\
\hline I make contact easily when I meet people* & $2.15(1.03)$ & $2.03(1.04)$ & 0.219 \\
\hline I often make a fuss about unimportant things & $1.38(0.88)$ & $1.35(0.85)$ & 0.504 \\
\hline I often talk to strangers* & $2.58(0.88)$ & $2.61(0.99)$ & 0.508 \\
\hline I often feel unhappy & $1.59(0.94)$ & $1.44(1.02)$ & 0.066 \\
\hline I am often irritated & $1.84(1.02)$ & $1.78(1.07)$ & 0.505 \\
\hline I often feel inhibited in social interactions & $2.03(1.02)$ & $1.85(1.01)$ & $0.035^{\star}$ \\
\hline I take a gloomy view of things & $1.86(0.97)$ & $1.72(0.97)$ & 0.112 \\
\hline I find it hard to start a conversation & $2.06(0.99)$ & $1.90(1.09)$ & 0.098 \\
\hline I am often in a bad mood & $1.48(0.92)$ & $1.38(0.91)$ & 0.253 \\
\hline I am a closed kind of person & $2.00(1.05)$ & $1.76(1.05)$ & $0.008^{*}$ \\
\hline I would rather keep other people at a distance & $2.20(0.92)$ & $2.03(0.99)$ & 0.092 \\
\hline I often find myself worrying about something & $2.22(0.97)$ & $1.99(1.10)$ & $0.018 \%$ \\
\hline I am often down in the dumps & $1.94(1.02)$ & $1.67(1.04)$ & $0.006^{*}$ \\
\hline When socializing, I don't find the right things to talk about & $1.99(0.91)$ & $1.85(0.99)$ & 0.191 \\
\hline Total & $27.37(9.07)$ & $25.35(9.49)$ & $<0.001$ * \\
\hline
\end{tabular}

Data are presented as mean (standard deviation). * Mann-Whitney $U$ test $P<0.05 *$ Reverse scored

\section{Assessment of Type D personality}

A Type D personality was identified in 152 participants $(67.6 \%)$ in the dropout group and in 138 participants (58.5\%) in the maintenance group (Table 4$)$. The difference was significant on the $2 \times 2$ chi-square test. Furthermore, the two groups significantly differed in negative affectivity $(P=0.039)$. Table 5 shows the details of the DS14 items. The proportions of respondents reporting that "I am a closed kind of person" and "I am often down in the dumps" were significantly different $(P<0.01)$.

\section{Discussion}

The present findings suggest a relationship between Type D personality and withdrawal from dental therapy. The most common reason for treatment dropout was "improvement of symptoms", followed by "busy with business or study". With regard to participant attitudes, many respondents in the dropout group had negative feelings about dental practice.

In previous studies of factors associated with dental therapy dropout, the participants were frequently outpatients who had a history of dental therapy withdrawal (22-24). However, few reports assessed participants who were not in a dental clinic at the time of data collection.
In the present study, participants were not currently undergoing dental therapy; the most common reason for discontinuing dental therapy was "improvement of symptoms". O'Shea et al. reported that "moving out" was the most common reason for interruption of dental therapy (22). However, Becker et al. reported that "stressful life events" was the most common reason for treatment withdrawal (23), while Kuhner and Raetzke reported that "laziness" was the most common reason, followed by "busy with business" (24). The results of the present study differed most from those of O'Shea and colleagues. This discrepancy might be attributable to differences in the patient populations. The participants in the previous study were patients who had visited a dental clinic after dropping out, while the present participants had not resumed dental therapy. As noted above, Becker et al. reported that "stressful life events" was the most common reason for dropping out from dental therapy, while Kuhner and Raetzke reported that "laziness" was the most common reason. These discrepancies might be attributable to cultural differences. The present participants were Japanese and might therefore have been more inclined to make vague comments and retreat to safe ground. It might have been difficult for them to offer 
reasons such as "stressful life events" or "laziness".

The two groups significantly differed in many of the question items related to respondent attitudes. A previous study found that visiting a dental clinic for a check-up was affected by a positive perception of oral health (25). This result was consistent with the present items "The image I associate with dental practice are "neat", "kind" or "refreshing" and "I think it is important to regularly visit a dental clinic". Tamaki et al. reported a correlation between regular dental check-ups and patient satisfaction and a strong association between regular dental check-ups and the technical competence of the dental hygienist (26). The present questionnaire item "I feel comfortable with the staff" was similar to an item in their previous study. Moreover, a correlation between regular dental checkups and the patient-dentist relationship was reported (27). Thus, past and present results suggest that maintaining a good relationship between clinic staff members and patients might help prevent dental treatment dropout. It is unclear how to establish a good relationship with patients who have a Type D personality; however, regular follow-up visits or troubleshooting by phone might help improve adherence (28).

To the authors' knowledge, this is the first study of the relationship between Type D personality and adherence to dental therapy. The results are similar to those for the original Marlatt relapse model (29), which was used to investigate the psychological state of patients who withdrew from therapy for drug or alcohol abuse. The patients with a strong negative affect had a high treatment dropout rate (29). The emotional and cognitive states of the present respondents with a Type D personality were quite similar to those of the patients in that earlier study. Moreover, a Type D personality was associated with low adherence to diabetic care (16-18). Thus, a Type D personality is likely to be associated with lower adherence to dental therapy. In addition, the present results show that respondents who reported a large number of treatment interruptions were more likely to have a Type D personality. Thus, people who frequently interrupt dental treatments may have a Type D personality and could have a high risk of diabetes-related disease.

The present study had some limitations, the most important of which is that the investigation was webbased. The participants were self-selected and registered with a web research office; thus, selection bias was unavoidable. Furthermore, the reliability of responses is a concern because of the high level of anonymity offered by the web-based approach. However, the internet penetration rate in this study was $83.0 \%$, and many Japanese use the internet (White paper Information and communications in Japan, 18, 65, Ministry of Internal Affairs and Communications, 2018.). Therefore, selection bias might have been lower than it would have been in the previous decade. Furthermore, web-based research allows for inappropriate respondents to be removed from the analysis, which improves response reliability $(30,31)$. The web research company used in this study routinely screens respondents.

In a previous study that used phone interviews of patients who withdrew from dental therapy, the present authors found it difficult to contact patients because of loss of contact (when patients moved) or respondent distrust of dental practice. This resulted in a follow-up rate of only $5.38 \%$. Thus, because of the difficulty in enrolling participants who had withdrawn from dental therapy, web-based research might be the only option. In the future, development of informatics technology might require patient information to be shared with other dental offices. Furthermore, information from patients who discontinue treatment may be tracked. This would facilitate study of the association between patient attitudes and cessation of dental therapy.

In conclusion, the present results indicate that a Type $\mathrm{D}$ personality is associated with withdrawal from dental therapy. The most common reason for withdrawing from dental therapy was improvement of symptoms, and the most common patient attitude was negative feelings about clinical dental practice.

\section{Acknowledgments}

The authors are grateful to the patients and staff members of the Sugiyama Dental Clinic and thank their colleagues for supporting the present study.

\section{Conflict of interest}

The authors declare that they have no competing interests.

\section{References}

1. Bahekar AA, Singh S, Saha S, Molnar J, Arora R (2007) The prevalence and incidence of coronary heart disease is significantly increased in periodontitis: a meta-analysis. Am Heart J 154, 830-837.

2. Awano S, Ansai T, Takata Y, Soh I, Akifusa S, Hamasaki T et al. (2008) Oral health and mortality risk from pneumonia in the elderly. J Dent Res 87, 334-339.

3. Chambrone L, Guglielmetti MR, Pannuti CM, Chambrone LA (2011) Evidence grade associating periodontitis to preterm birth and/or low birth weight: I. A systematic review of prospective cohort studies. J Clin Periodontol 38, 795-808.

4. Iwasaki M, Taylor GW, Nesse W, Vissink A, Yoshihara A, Miyazaki H (2012) Periodontal disease and decreased kidney function in Japanese elderly. Am J Kidney Dis 59, 202-209. 
5. Iwasaki M, Taylor GW, Manz MC, Kaneko N, Imai S, Yoshihara A et al. (2012) Serum antibody to Porphyromonas gingivalis in chronic kidney disease. J Dent Res 91, 828-833.

6. Axelsson P, Lindhe J (1981) Effect of controlled oral hygiene procedures on caries and periodontal disease in adults. Results after 6 years. J Clin Periodontol 8, 239-248.

7. Miyamoto T, Kumagai T, Lang MS, Nunn ME (2010) Compliance as a prognostic indicator. II. Impact of patient's compliance to the individual tooth survival. J Periodontol 81, 1280-1288.

8. Renvert S, Persson GR (2004) Supportive periodontal therapy. Periodontol 2000 36, 179-195.

9. McCracken G, Asuni A, Ritchie M, Vernazza C, Heasman P (2017) Failing to meet the goals of periodontal recall programs. What next? Periodontol 2000 75, 330-352.

10. Denollet J (2005) DS14: standard assessment of negative affectivity, social inhibition, and Type D personality. Psychosom Med 67, 89-97.

11. Denollet J, Conraads VM. (2011) Type D personality and vulnerability to adverse outcomes in heart disease. Cleve Clin J Med 78, 13-19.

12. Denollet J, Vaes J, Brutsaert DL (2000) Inadequate response to treatment in coronary heart disease: adverse effects of type $\mathrm{D}$ personality and younger age on 5 -year prognosis and quality of life. Circulation 102, 630-635.

13. Denollet J, Sys SU, Stroobant N, Rombouts H, Gillebert TC, Brutsaert DL (1996) Personality as independent predictor of long-term mortality in patients with coronary heart disease. Lancet 347, 417-421.

14. Denollet J, Martens EJ, Nyklícek I, Conraads VM, de Gelder B (2008) Clinical events in coronary patients who report low distress: adverse effect of repressive coping. Health Psychol 27, 302-308.

15. Wu JR, Moser DK (2014) Type D personality predicts poor medication adherence in patients with heart failure in the USA. Int J Behav Med 21, 833-842.

16. Milicevic R, Jaksic N, Aukst-Margetic B, Jakovljevic M (2015) Personality traits and treatment compliance in patients with type 2 diabetes mellitus. Psychiatr Danub 27, Suppl 2, 586-589.

17. Conti C, Carrozzino D, Patierno C, Vitacolonna E, Fulcheri M (2016) The clinical link between Type D personality and diabetes. Front Psychiatry, June 21, doi.org/10.3389/ fpsyt.2016.00113.

18. Li X, Zhang S, Xu H, Tang X, Zhou H, Yuan J (2016) Type D personality predicts poor medication adherence in Chinese patients with type 2 diabetes mellitus: a six-month follow-up study. PLoS One, Feb 19, doi.org/10.1371/journal. pone. 0146892 .
19. Loosman WL, de Jong RW, Haverkamp GLG, van den Beukel TO, Dekker FW, Siegert CEH et al. (2018) The stability of Type D personality in dialysis patients. Int J Behav Med 25, 85-92.

20. Mizutani S, Ekuni D, Yamane-Takeuchi M, Azuma T, Taniguchi-Tabata A, Tomofuji T et al. (2018) Type D personality and periodontal disease in university students: a prospective cohort study. J Health Psychol 23, 754-762.

21. Kato T, Sugiyama S, Makino M, Naito T (2016) A qualitative study on the background of long-term maintenance patients at a private Japanese dental clinic. BMC Oral Health, Apr 1, doi. org/ 10.1186/s12903-016-0203-2.

22. O'Shea RM, Corah NL, Ayer WA (1986) Why patients change dentists: practitioners' views. J Am Dent Assoc 112, 851-854.

23. Becker BE, Karp CL, Becker W, Berg L (1988) Personality differences and stressful life events. Differences between treated periodontal patients with and without maintenance. J Clin Periodontol 15, 49-52.

24. Kühner MK, Raetzke PB (1989) The effect of health beliefs on the compliance of periodontal patients with oral hygiene instructions. J Periodontol 60, 51-56.

25. Afonso-Souza G, Nadanovsky P, Chor D, Faerstein E, Werneck GL, Lopes CS (2007) Association between routine visits for dental checkup and self-perceived oral health in an adult population in Rio de Janeiro: the Pró-Saúde sudy. Community Dent Oral Epidemiol 35, 393-400.

26. Tamaki Y, Nomura Y, Nishikawara F, Motegi M, Teraoka K, Arakawa $\mathrm{H}$ et al. (2005) Correlation between patient satisfaction and dental clinic credibility in regular dental check-ups in Japan. J Oral Sci 47, 97-103.

27. Gürdal P, Cankaya H, Onem E, Dinçer S, Yílmaz T (2000) Factors of patient satisfaction/dissatisfaction in a dental faculty outpatient clinic in Turkey. Community Dent Oral Epidemiol 28, 461-469.

28. Maschauer EL, Fairley DM, Riha RL (2017) Does personality play a role in continuous positive airway pressure compliance? Breathe (Sheff) 13, 32-43.

29. Hendershot CS, Witkiewitz K, George WH, Marlatt GA (2011) Relapse prevention for addictive behaviors. Subst Abuse Treat Prev Policy, Jul 19, doi.org/10.1186/1747597X-6-17.

30. Berinsky AJ, Margolis MF, Sances MW (2014) Separating the shirkers from the workers? making sure respondents pay attention on self-administered surveys. Am J Pol Sci 58, 739-753.

31. Chandler J, Mueller P, Paolacci G (2014) Nonnaïveté among Amazon Mechanical Turk workers: consequences and solutions for behavioral researchers. Behav Res Methods 46, 112-130. 